\title{
Complete Remission and Long Term Survival in Recurrent Malignant Glioblastoma Treated with Fotemustine Monotherapy: A Case Report*
}

\author{
Carmine Cerullo ${ }^{1 \#}$, Carla Fonte ${ }^{1}$, Andrea Muto ${ }^{1}$, Agnese Vannini ${ }^{1}$, Mattia Rediti ${ }^{1}$, Sheila Rangan ${ }^{1}$, \\ Lorenzo Bordi ${ }^{2}$, Bruno Neri ${ }^{1}$
}

${ }^{1}$ Department of Oncology_Center of Experimental and Clinical Oncology, University of Florence, Florence, Italy; ${ }^{2}$ Interventional Neuroradiology, Florence, Italy.

Email: "c.cerullo@libero.it, bruno.neri@unifi.it

Received January $20^{\text {th }}, 2013$; revised February $22^{\text {nd }}, 2013$; accepted March $2^{\text {nd }}, 2013$

Copyright $\odot 2013$ Carmine Cerullo et al. This is an open access article distributed under the Creative Commons Attribution License, which permits unrestricted use, distribution, and reproduction in any medium, provided the original work is properly cited.

\begin{abstract}
Glioblastoma multiforme (GBM) is the most malignant primary brain tumor. Conventional therapies are considered palliative and long-term progression-free survival remains low for most GBM patients even after surgical excision, concomitant radiotherapy and/or chemotherapy with nitrosoureas or Temozolomide. We report the case of a 47-year-old man who presented worsening headache over a month, accompanied by episodes of morning vomiting and hyposthenia of the left hemisoma. Cerebral tomographic scan revealed an expansive process on the right frontal site. Following surgical resection, the patient was diagnosed with diffusely infiltrating GBM. After surgery, the patient underwent radiotherapy and chemotherapy with Temozolomide for 6 months. Eleven months later, the patient underwent a second-look surgery with excision of a new right superior frontal nodule. This time the patient refused both radio- and chemotherapy and underwent follow-up only. After 12 months he underwent a new craniotomy for the excision of a third GBM relapse. Following resection, the patient agreed to be treated for 6 months with fotemustine (FTM) 100 $\mathrm{mg} / \mathrm{m}^{2}$ intravenously every 4 weeks as chemotherapy. After 3 months of treatment the patient underwent a full physical examination and diagnostic monitoring of the tumor using Magnetic Resonance Imaging (MRI) of the brain and whole body Computerised Tomography (CT). During FTM treatment, the most frequent, but reversible toxic effect was hematological grade 2 anemia and thrombocytopenia but no other grade $>2$ toxicity was recorded, so there was no reduction or delay in treatment. Presently, after 8 years of follow-up, the patient is in excellent health and has no evidence of intracranial disease recurrence. In light of this case, post-surgical FTM treatment seems to represent an interesting well-tolerated treatment possibility in patients with recurrent malignant GBM of the brain, given that there are very few reports of such a remission in the literature.
\end{abstract}

Keywords: Fotomustine; Glioblastoma Multiforme; Recurrence; Treatment; Survival

\section{Introduction}

Meningiomas, glioblastomas and astrocytomas constitute over half of all central nervous system (CNS) tumors. Glioblastoma multiforme is a highly malignant astrocytic tumor that accounts for $80 \%$ of all the malignant gliomas. It is a neoplasm occurring mainly in adult age with peak incidence in individuals between 60 and 70 years of age, with prevalence in males [1]. Malignant gliomas display an invasive phenotype with the tendency for locore-

*Disclosure: The authors report no conflict of interest concerning the materials or methods used in this study or the findings specified.

\#Corresponding author. gional spread. By following the joints and nerve fiber sheaths they can extend to the encephalon, invade the basal nuclei, the internal capsule and, through the corpus callosus, the controlateral hemisphere. For this reason it is difficult to cure these tumors [2]. The great abundance of newly formed vessels within the tumor is responsible for the frequent occurrence of intratumoral hemorrhage and of the presence of vast areas of necrosis secondary to vascular thrombosis. With extremely rare exceptions, these tumors do not spread outside the CNS, however, their unfavorable evolution depends on the effect of the intracranial mass which gradually increases intracranial 
pressure, causing compression syndromes, coma and death.

The treatment program is multidisciplinary [3]: surgical resection is the first step, followed by radiotherapy in the tumor invasion area. Adjuvant chemotherapy with temozolomide (TMZ) after radical surgery and radio-therapy can be effective in prolonging patient survival and keeping disease progression at abeyance [4,5]. However, despite combined treatment, median survival ranges from 12 to 15 months for GBM and from 2 to 5 years for anaplastic gliomas [6]. Second debulking surgery alone does not seem to improve the prognosis, as almost all patients die within 6 months after the second surgery. So, the problem of unsatisfactory local control of recurrence continues to exist. Such a dismal prognosis is mainly due to the rapid onset of radio- and/or chemoresistance as well as to the limited therapeutic options available for GBM recurring after standard treatment. However, recent clinical studies have shown that fotemustine (FTM) seems to represent an interesting well-tolerated treatment possibility in pretreated patients with recurrent malignant GBM [7-9].

FTM is a third-generation chloroethylnitrosourea characterized by a phosphoalanine carrier group grafted onto the nitrosourea radical, which confers high lipophilicity and better cell membrane penetration [10].

In this study we used magnetic resonance imaging (MRI) to describe the response of a 47-year-old male with GBM, who had been pretreated and suffered a third relapse who was treated for 6 months with FTM.

\section{Case Report}

In April, 2003 a 47-year-old male presented to the AOUCareggi Emergency Unit because of worsening headache which he had suffered for over a month, accompanied by episodes of morning emesis and hyposthenia of the left hemisoma. He underwent a brain computerized tomography (CT) scan (Figure 1) that showed an expansive process in the right frontal region. After a further MRI confirmation and after a complete radiological survey to exclude other localizations, he underwent craniotomy with excision of the lesion in June, 2003. The definitive histological analysis of the lesion showed a diffusely infiltrating GBM. Immediately after surgery, the patient underwent adjuvant radiochemotherapy with oral TMZ for 2 months and home therapy with dintoina was started.

A brain CT performed during the routine follow-up in April 2004 (Figure 2) revealed a new nodule on the right frontal pole, whose image contrast characteristics were consistent with a tissue nodule in the superior polar frontal region, compatible with GBM recurrence without clinical evidence. Therefore, in May 2004, the patient underwent a second-look surgery with excision of the right superior frontal nodule. Both the intraoperative and

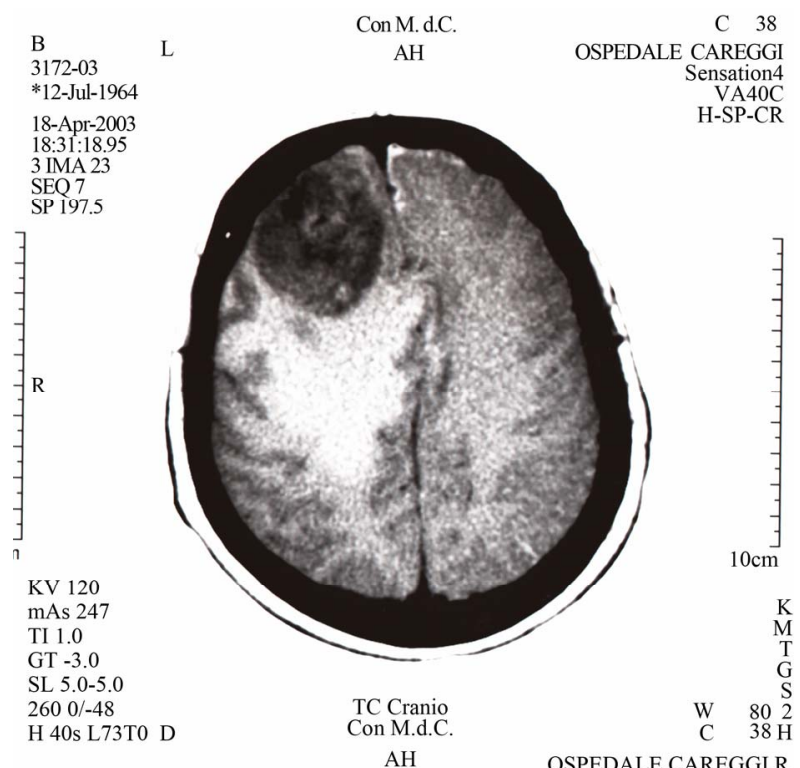

Figure 1. Brain CT 2003.

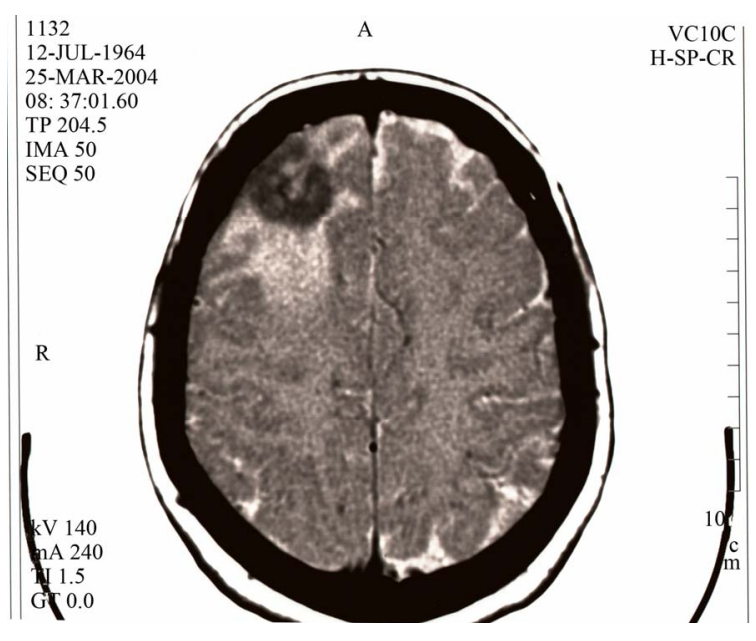

Figure 2. Brain CT 2004.

definitive histological examination confirmed the neoplastic nature of the lesion, consistent with GBM recurrence. After surgery the patient refused further TMZ chemotherapy and was only followed-up. A year later, during follow-up examination, the brain MRI findings revealed a further change, with the appearance of a new lesion at the site of the previous surgical excision for the third time. The patient underwent a new craniotomy to excise the second relapse of GBM on the right frontal site. After surgery, the patient was referred for the first time to the Oncology Outpatient Clinic of the Center of Experimental and Clinical Oncology where we managed to convince him to undergo intravenous chemotherapy with FTM as post-surgical adjuvant treatment. Subsequently, from June, 2005 until January, 2006, the patient was administered adjuvant chemotherapy accord- 
ing to the following schedule: Days 1 - 8: FTM $100 \mathrm{mg} / \mathrm{m}^{2}$ i.v. (as induction), Day 30: FTM $100 \mathrm{mg} / \mathrm{m}^{2}$ i.v. every 30 days for 6 cycles.

During the entire treatment period, the general and hematological toxicity were well controlled. WHO grade toxicity was always $<3$ without the need for hospitalization or for requiring reduction of the FTM dose during treatment. This probably was also related to the good physical condition of the patient, the absence of comorbidities, and relatively young age.

In March 2005, at the end of chemotherapy we carried out a full check-up of the patient which indicated he had excellent general condition and as confirmed by imaging studies including brain MRI that revealed no signs of relapse.

Today, 7 years after the last GBM recurrence, the patient is still in good health and has no clinical manifestations related to the disease or the treatments he underwent. Since March 2005, brain MRI has been regularly performed every year and has not shown any evidence of GBM recurrence (Figures 3 and 4).

\section{Discussion}

Glioblastomas, the most malignant astrocytic neoplasms, are composed of poorly differentiated neoplastic astrocytes with areas of vascular hyperplasia and zones of coagulation necrosis. GBM is a relatively frequent intracranial neoplasm in adults (12\% - $20 \%$ of all intracranial tumors) [11]. The treatment of GBM is still a challenge, and despite first-line therapy consisting of surgery, radiotherapy and TMZ as adjuvant chemotherapy, the tumor invariably recurs, allowing median survival of only 9 - 12 months [9]. To date, little data are available regarding GBM patients with more than 5 years' survival, with percentages varying from $2 \%-5 \%[12,13]$. In case of relapse, second debulking surgery alone does not seem to improve the

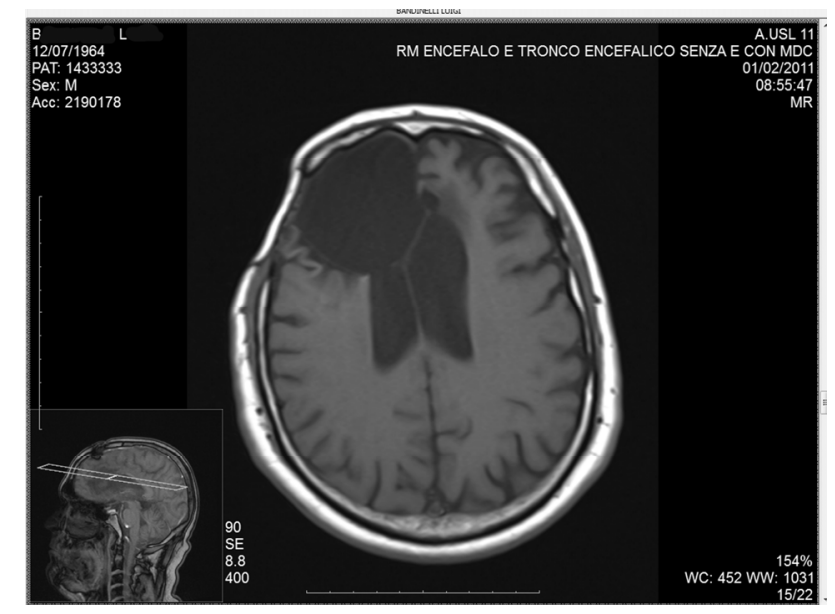

Figure 3. Brain MRI 2011.

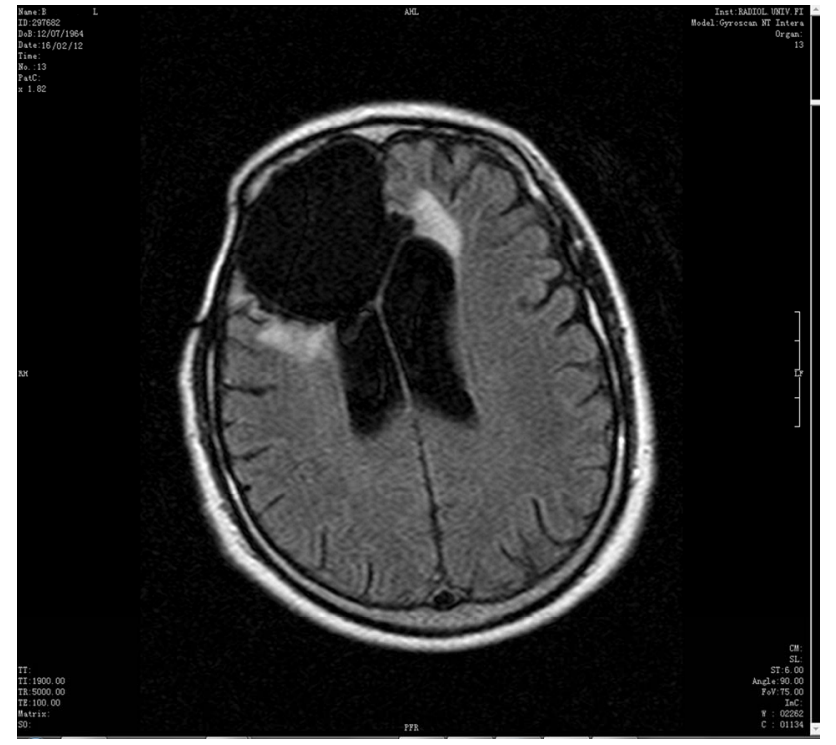

Figure 4. Brain MRI 2012.

prognosis. At the same time, there are no clearly established chemotherapy regimens for the treatment of recurrent GBM.

In recent years there have been several agents and chemotherapy regimens used in the treatment of recurrent GBM but all with very only short progression-free intervals and a median survival rarely exceeding 6 months. A few phase II studies have reported about second-line chemotherapy in patients previously treated with TMZ either in the adjuvant setting [14] or at first recurrence [15].

FTM (diethyl-1-3-(2-chloroethyl)-3nitrosureido) ethylphosphonate) is a liposoluble nitrosourea. It consists structurally of a nitrosourea radical attached to an aminophosphonic acid group, as a carrier, to increase its ability to penetrate the blood-brain barrier and through cell membranes [16]. Data on the outcome of FTM administration for progressing/relapsing GBM used as secondline treatment, report median survival of 21 - 27 months from the first diagnosis of the disease $[8,16]$. This drug, using the conventional schedule of $100 \mathrm{mg} / \mathrm{m}^{2}$ intravenously every week for 3 consecutive weeks followed by a 5-week rest period and maintenance treatment with one infusion every 3 weeks [17], has shown hematological toxicity as the most relevant side effect, being reported in more than $30 \%$ of pretreated chemo- therapy patients. A previous phase II study in ocular melanoma patients has demonstrated that FTM administered according to the schedule we used, had reduced hematological toxicity while maintaining favorable results in terms of response [18].

In this case report, we describe the management of highly invasive, recurrent GBM, non-responsive to adju- 
vant TMZ-chemotherapy in a young patient where rescue treatment with FTM administered every 30 days after the induction doses was able to fully control the antiblastic activity without provoking unmanageable hematological toxicity. This result strongly suggests that FTM should be tested in an adequate randomized study to elucidate its possible role in a new adjuvant setting as first-line treatment of GBM. We would like to emphasize that to date, February 2012, 8 years have elapsed since the first manifestation of the disease and our patient has now arrived to age 56 without showing any signs of illness.

\section{Acknowledgements}

The authors acknowledge the assistance of Prof. Grazia Cini for editing the manuscript.

Funding for this study was provided by the "Associazione Toscana Ricerche e Cure Oncologiche" FlorenceItaly.

\section{REFERENCES}

[1] T. A. Dolecek, J. M. Propp, N. E. Stroup and C. Kruchko, "CBTRUS Statistical Report: Primary Brain and Central Nervous System Tumors Diagnosed in the United States in 2005-2009," Neuro-Oncology, Vol. 14, No. 5, 2012, pp. v1-v49. doi:10.1093/neuonc/nos218

[2] R. Stupp, M. E. Hegi and M. J. Van den Bent, et al., "Changing Paradigms; an Update on the Multidisciplinary Management of Malignant Glioma,” Oncologist, Vol. 11, No. 2, 2006, pp. 165-180. doi:10.1634/theoncologist.11-2-165

[3] R. Stupp and F. Roila, "ESMO Giudelines Working Group. Malignant Glioma: ESMO Clinical Recommendations for Diagnosis, Treatment, and Follow-Up,” Annals of Oncology, Vol. 20 Suppl 4, 2009, pp. 126-128. doi:10.1093/annonc/mdp151

[4] H. A. Fine, K. B. Dear, J. S. Loeffler, et al., "Meta-Analysis of Radiation Therapy with and without Adjuvant Chemotherapy for Malignant Gliomas in Adults," Cancer, Vol. 71, No. 8, 1993, pp. 2585-2597. doi:10.1002/1097-0142(19930415)71:8<2585::AID-CNC $\underline{\text { R2820710825>3.0.CO;2-S }}$

[5] L. A. Stewart, "Chemotherapy in Adult High-Grade Glioma: A Systematic Review and Meta-Analysis of Individual Patient Data from 12 Randomized Trials," Lancet, Vol. 359, No. 9311, 2002, pp. 1011-1018. doi:10.1016/S0140-6736(02)08091-1

[6] P. Y. Wen and S. Kesari, "Malignant Gliomas in Adults," The New England Journal of Medicine, Vol. 359, No. 5, 2008, pp. 429-507. doi:10.1056/NEJMra0708126

[7] A. Fabi, G. Metro, M. Russillo, et al., "Treatment of Recurrent Malignat Gliomas with Fotemustine Monotherapy: Impact of Dose and Correlation with MGMT Promoter
Methylation,” BMC Cancer, Vol. 9, 2009, pp. 101-105. doi:10.1186/1471-2407-9-101

[8] M. G. Fabrini, G. Silvano, I. Lolli, et al., “A Multi-Istitutional Phase II Study on Second-Line Fotemustine Chemotherapy in Recurrent Glioblastoma," Journal of NeuroOncology, Vol. 92, No. 1, 2009, pp. 79-86. doi:10.1007/s11060-008-9739-6

[9] R. Addeo, M. S. De Santi, S. Del Prete, et al., "Fotemustine and Recurrent Glioblastoma: Possible New Opportunities for an Old Drug," Cancer Chemotherapy and Pharmacology, Vol. 64, No. 5, 2009, pp. 863-866. doi:10.1007/s00280-009-1086-6

[10] A. Meulemans, B. Giroux, P. Hannoun, et al., "Comparative Diffusion Study of Two Nitrosoureas: Carmustine and Fotemustine in Normal Rat Brain, Human and Rat Brain Biopsies,” Chemotherapy, Vol. 37, No. 2, 1991, pp. 86-92. doi:10.1159/000238838

[11] M. Salcman, "Glioblastoma Multiforme and Anaplastic Astrocytoma,” In: A. H. Kaye and Er. Laws Jr., Eds., Brain Tumors: An Encyclopedic Approach, Churchill Livingstone, London, pp. 494-523.

[12] J. N. Scott, N. B. Rewcastle, P. M. Brasher, et al., "Which Glioblastoma Multiforme Patients Will Become a LongTerm Survivor? A Population-Based Study," Annals of Neurology, Vol. 46, No. 2, 1999, pp. 183-188. doi:10.1002/1531-8249(199908)46:2<183::AID-ANA7>3 $.0 . \mathrm{CO} ; 2-7$

[13] R. E. Mc Lendon and E. C. Halperin, "Is the Long-Term Survival of Patients with Glioblastoma Multiforme Overstated?” Cancer, Vol. 98, No. 8, 2003, pp. 1745-1753. doi:10.1002/cncr.11666

[14] R. Stupp, W. P. Mason, M. J. Van den Bent, M. Weller, et al., "Radiotherapy plus Concomitant and Adjuvant Temozolomide for Glioblastoma," The New England Journal of Medicine, Vol. 352, No. 10, 2005, pp. 978-996. doi:10.1056/NEJMoa043330

[15] M. Mousseau, P. Swiercz, M. Rougny, et al., "Fotemustine in Recurrent Supratentorial Malignant Gliomas," Drugs of Today, Vol. 32, Suppl. E, 1996, pp. 43-50.

[16] S. Scoccianti, B. Detti, A. Sardaro, et al., "Second-Line Chemotherapy with Fotemustine in Temozolomide-Pretreated Patients with relapsing Glioblastoma: A Single Institution Experience,” Anti-Cancer Drugs, Vol. 19, No. 6, 2008, pp. 613-620. doi:10.1097/CAD.0b013e3283005075

[17] M. Frenay, B. Giroux, S. Khoury, et al., "Phase II Study of Fotemustine in Recurrent Supratentorial Malignant Gliomas,” European Journal of Cancer, Vol. 27, No. 7, 1991, pp. 852-856. doi:10.1016/0277-5379(91)90133-X

[18] P. Pinzani, C. Mazzini, B. Neri, et al., "Tyrosinase mRNA Levels in Blood Uveal Melanoma Patients: Correlation between the Number of Circulating Tumor Cells and Tumor Progression,” Melanoma Research, Vol. 20, No. 4 2010, pp. 303-310. doi:10.1097/CMR.0b013e32833906e3 